\title{
Del «efecto Simmel» y la autopersuasión: la teoría cognitivista de las creencias de R. Boudon
}

\author{
Ángeles Lizón
}

\section{Resumen}

La preocupación por las ceencias y las convicciones colectivas es una cuestión clásica de la sociología fundamental. R. Boudon se acerca a esta reflexión desde una perspectiva interdisciplinaria. El «modelo Simmel» es el eje de su argumentación que se describe en el artículo, haciéndola converger en su teoría "cognitivista» de las creencias.

Palabras clave: teoría cognitivista, «modelo Simmel», teoría racional de las creencias, creencias colectivas.

Abstract. From "Simmel effect» and the autoconviction: The cognitive theory of believes of R. Boudon

The interest for colective believes and convictions is a classic question of Sociology. R. Boudon thinks about this question from a interdisciplinar perspective. The "Simmel model» is the main idea of his argumentation.

Key words: cognitive theory, "Simmel model», collective believes, rational theory of believes.

\section{Sumario}

1. Las teorías de las creencias: una tipología de la tradición heredada

2. No somos tan racionales: la argumentación contra el "pensamiento prelógico» de Lévy-Bruhl

3. Tampoco somos tan irracionales: la argumentación contra la tesis de R.A. Shweder
4. $\mathrm{El}$ «modelo Simmel»

5. Esbozo de un teoría racional de las creencias

6. Mejor aún que racional

Referencias bibliográficas 
En L'Art de se persuader des idées douteuses, fragiles ou fausses $\mathrm{R}$. Boudon mezcla, de forma provocativa e interesante, al menos tres ideas con implicaciones nada desdeñables para la teoría de la racionalidad. En primer lugar, incorpora a la discusión el problema de Kahneman y Tversky o de las heurísticas problemáticas y hace de la evidencia experimental de los psicólogos cognitivos sobre los fallos sistemáticos del razonamiento intuitivo una cuestión central. En segundo lugar, tema también muy traído por la psicología cognitiva, considera el problema de J.S. Mill o de los juicios que envuelven una operación intelectual que toma por suficiente información insuficiente. Y, finalmente, se centra en la teoría de la inferencia natural de George Simmel y reconstruye un mecanismo cognitivo por medio del cual pueden describirse procesos de persuasión y adhesión a ideas erróneas sustentadas sobre inferencias válidas contaminadas por implícitos no conscientes que deforman las consecuencias. En el cuerpo central del libro, estas tres cuestiones, todas de alguna forma relacionadas con el problema de la inferencia inductiva, son ilustradas con diversos casos en los que sesgos cognitivos, epistemológicos o lingüísticos llevan a la adhesión a creencias en ideas frágiles, dudosas o falsas. En la discusión de cada una de estas instancias, el autor perfila e ilustra lo que él ha dado en llamar el "modelo Simmel», un mecanismo cognitivo de autopersuasión por el que «una idea [dudosa o falsa] fundada sobre una argumentación válida tiene más posibilidad de imponerse que una idea no fundada o mal fundada» (ibídem: 176).

Aunque los procesos de persuasión pueden también darse por otros procedimientos $^{1} \mathrm{y}$, lo más frecuente, es que éstos respondan directamente a factores motivacionales o afectivos, en presencia de este mecanismo la autopersuasión suele ser resultado de una argumentación correcta sustentada en principios comúnmente sostenidos e implícitamente considerados como legítimos. De esta forma, el sujeto cuenta con buenas razones para adherirse a creencias dudosas, inciertas o falsas. La descripción de este mecanismo o «modelo Simmel» constituye aquí el eje de la argumentación de Boudon, que se expondrá a continuación haciéndola convergir en su — tentativateoría «cognitivista» de las creencias, a la que se le plantearán algunos interrogantes.

La preocupación por las creencias y las convicciones colectivas es una cuestión clásica de la sociología fundamental que ha cobrado renovado interés en las últimas décadas. Mérito de Boudon es no ya haberse embarcado a fondo en esta reflexión, sino haberlo hecho desde una perspectiva claramente interdisciplinaria. Es evidente que, dado el estadio actual de muchas de las disciplinas colindantes con la sociología, no se puede seguir trabajando en solitario en aras de cualquier pretendido ideal de «autonomía». El testimonio de Boudon señala cómo el trabajo del sociólogo, sea cual sea la conjetura final

1. Boudon (1990: 48) cita especialmente los trabajos de los teóricos de la argumentación Toulmin y Perelman y su tesis de la argumentación jurídica: se pueden tener buenas razo nes sin que éstas sean demostrativas. Para más cfr. (ibídem: 48-56). 
a la que quiera o pueda llegar, no puede competentemente hacerse ignorando los avances de las ciencias que le incumben, y ha de tener, indispensablemente, una óptica inter e intradisciplinaria ${ }^{2}$.

\section{Las teorías de las creencias: una tipología de la tradición heredada}

\section{En Les puissances qui nous portent à consentir Boudon (1990: cap. 1) hace al} menos tres cosas. En primer lugar, intenta establecer una primera tipificación de las teorías de formación de creencias, sienta luego la necesidad de recuperar la idea de la universalidad de la lógica y, finalmente, se sirve de ello para argumentar a favor de las nuevas tendencias que apuestan por posponer los factores motivacionales y priorizar las razones cognitivas en la explicación de las mismas.

Ya de partida, la idea de sostener las razones como causas de las creencias («el sujeto se muestra dispuesto o se siente impelido a conferir a la verdad del enunciado $Y$ la causa de su creencia en $Y_{\Perp}$ : Boudon, 1990: 22), un clásico dic tum weberiano, tiene decididos defensores y claros detractores. De hecho, las aportaciones heredadas de las teorías socioantropológicas y aun psicológicas de forma prioritaria parecen poner el énfasis precisamente en lo contrario, a saber, el carácter irracional (las causas no tienen el estatuto de razones) de los factores determinantes de creencias. Sin pretender construir una tipología exhaustiva, Boudon $(1990,1995,1996)$ identifica distintos conjuntos de explicación de creencias que han cristalizado como las tendencias centrales de esta tradición acumulada. Transcrita libremente para los propósitos expositivos de este ejercicio, tal tipología puede reconstruirse en un esquema simple que se recoge en el cuadrocuadro 1. En él se condensan las posturas nucleares de la tradición heredada para la que, a excepción de las objetivamente fundadas (1), las creencias o los fenómenos de convicción atienden o (2) a factores cognitivos que no tienen el estatus de razones, (3) a factores afectivos o motivacionales o (4) se conciben como directamente tributarias de causas culturales o biológicamente evolucionarias.

Según las posturas tipificadas en el cuadro, para el primer conjunto expli -

2. Este sentimiento de «indispensabilidad» no es de hecho una novedad. La idea es tan vieja al menos como la intuición de Pareto, que ya en la introducción a sus escritos sociológicos decía: «La psicología está, evidentemente, en la base de la economía política y, en general, de todas las ciencias sociales. Quizá llegará un día en el que podremos deducir, a partir de los principios de la psicología, las leyes de la ciencia social [...], pero nos encontramos todavía bastante lejos de este estado de cosas y hemos de tomar otro camino». La novedad, lo que realmente parece apremiarnos hoy a la integración interdisciplinaria son los nuevos e interesantes avances logrados en los últimos años por la neurociencia, los estudios de la mente y de la inteligencia artificial, las ciencias cognitivas, y los - ya imparables- desarrollos de la genética, la ingeniería biológica y la biología y la psicología evolucionarias. Como afirma E.O. Wilson (1998: 22) en un reciente escrito: «hoy no se puede adquirir una perspectiva equilibrada estudiando las disciplinas a retazos, sino a través de la búsqueda de consiliencia entre ellas». 
Cuadro 1. Núcleo central de las distintas teorías de formación de creencias.

\begin{tabular}{|l|c|c|}
\hline \multicolumn{1}{|c|}{$\begin{array}{c}\text { Factores que } \\
\text { explican }\end{array}$} & «Razones» son causas: & Causas que «no-razones»: \\
\hline $\begin{array}{l}\text { Referidas a } \\
\text { factores cognitivos: }\end{array}$ & $\begin{array}{l}\text { 1. Explicar la creencia es hacer- } \\
\text { la comprensible dando cuen- } \\
\text { ta de las razones que se tie- } \\
\text { nen para creer en ella. }\end{array}$ & $\begin{array}{r}\text { 2. La explicación de la creencia } \\
\text { se apoya sobre causas que no } \\
\text { tienen el estatus de razones. }\end{array}$ \\
\hline Factores no-cognitivos: & $\begin{array}{l}\text { 4. Las razones que se da el suje- } \\
\text { to son tributarias de (a) cau- } \\
\text { sas culturales o (b) biológicas } \\
\text { evolucionarias. }\end{array}$ & $\begin{array}{l}\text { 3. Causas afectivas (accesibles o } \\
\text { no a la observación). }\end{array}$ \\
\hline
\end{tabular}

car la creencia de un grupo es hacerla comprensible, dando cuenta de las razo nes que tienen sus miembros para creer en ellas. En este caso, la explicación de las creencias se fundamenta sobre factores cognitivos identificados con «razones» y la tradición sociológica encuentra básicamente en Weber una importante representación de la tendencia (teorías de tipo 1). Para el segundo grupo, en cambio, la explicación de las creencias obedece a factores cognitivos, no-afectivos, aunque no identificables con razones (teorías de tipo 2). Trabajos representativos de esta tendencia son los clásicos estudios de LévyBruhl sobre las creencias mágicas. En ellos el autor da por buena la idea de que su ley de participación ${ }^{3}$ y la mentalidad-primitiva ${ }^{4}$ imponen al sujeto una suerte de pensamiento prelógico. El primitivo obedece a reglas de razonamiento distintas de las nuestras, reglas culturalmente variables, asociadas a los distintos estadios de desarrollo sociocultural y a las que el sujeto no puede enfrentarse con distancia crítica. Dada la afinidad de interpretación de muchos psicólogos cognitivistas contemporáneos con este tipo de explicación, su revisión cobrará particular relevancia en esta exposición. Las explicaciones de las creencias que caen en la tercera casilla (teorías de tipo 3) responden a factores directamente motivacionales y, en la más estricta tradición paretiana, se presentan como efecto directo del sentimiento (como residuos). Con una concepción esencialmente motivacional, aquí las razones que pueda creer darse el sujeto sólo tienen una función "racionalizadora» (Freud) o un carácter abiertamente «ilusorio» (Pareto). Contemporáneos como Aronson (1968) se han encargado de mantener viva esta idea de un sujeto social «razonador» pero no necesariamente «razonable». Finalmente, en lo que respecta al último conjunto, las razones aparecen como claramente vicarias. Se presentan como efectos de otros procesos y son abiertamente consideradas como tributarias de causas biológicas o culturales evolucionarias. La tradición más fuerte en este caso ha

3. Lévy-Bruhl (1922). Se cita la edición de PUF (1960: 77).

4. Ibídem: pág. 31 . 
sostenido la idea de que las razones vienen impuestas al sujeto social por internalización o a través de procesos de socialización (teorías de tipo 4).

Las explicaciones de tipo 2 y 3 «son explicaciones «irracionales» y comparten un mismo postulado inicial, a saber, que las creencias del sujeto social se explican no por las razones que éste se da o se puede dar, sino por causas de otra naturaleza. Podrían también llamarse «ilusionistas» en tanto que las razones que se da el sujeto se tratan como meras ilusiones (1995: 62). No es que llanamente se pretenda subestimar la importancia que los esquemas de corte motivacional o afectivo pueden tener en la explicación de determinadas creencias 5 . Pero, una cosa es reconocer la existencia de tales procesos, e incluso admitir que tienen un papel importante en la formación de convicciones y en la adhesión a creencias, y otra suponer que todas las creencias no objetivamente fundadas tienen un origen exclusivamente afectivo. Convencido de que las ventajas que se experimentan al transcribir estas explicaciones a versiones más ajustadas a las explicaciones de tipo I, la tesis de Boudon se perfilará, pues, exactamente en sentido contrario. En ello no trabaja en solitario. Aunque de forma no necesariamente interdependiente, la tendencia a recuperar los factores cognitivos ha sido avalada por muchas otras contribuciones contemporáneas que provienen del campo de la psicología cognitiva y evolucionaria, la filosofía del conocimiento, la sociología y la historia de la ciencia, además de las reflexiones que la propia teoría económica ha hecho a su concepto estándar de «racionalidad». Estos desarrollos en aluvión han alentado intentos de recuperación del clásico dictum weberiano: para dar cuenta de una creencia -o de una acción-, siempre se ha de intentar encontrar las razones de la misma. Éste es expresamente el empeño de L'Art de se persuader, trabajo en el que Boudon emprende sistemáticamente el camino de la conciliación entre las convicciones objetivamente fundadas y aquéllas sustentadas en ideas frágiles, dudosas y aun falsas.

\section{No somos tan racionales: la argumentación contra el «pensamiento prelógico» de Lévy-Bruhl}

Aunque Weber le habría ofrecido una postura más constante y explícita ${ }^{6}$, su teoría de la magia le resulta demasiado comprimida. Boudon (1990: 30-47) opta por decantarse por la explicación de las creencias mágicas de E. Durk-

5. Boudon (1995: 62) alude aquí al caso del hombre de ciencia que tiene una buena corazonada y se dedica intensamente a contrastarla, tomándose muy a pecho ciertos hechos que directamente le incumben y descuidando o negligiendo otros que no considera pertinentes. A la postre, su propia vehemencia puede llevarle a adherirse a una creencia dudosa o falsa.

6. Boudon (ibídem: 44) señala cómo para Weber «explicar» las creencias religiosas de un grupo era siempre dar cuenta de las razones que sus miembros tienen para creer en ellas. Lo mismo pasa en la mayoría de sus análisis en materia de sociología de las religiones. Sometiendo algunos de ellos a su test lingüístico (ibid: 43), los ejemplos entresacados resultan muy eficaces: «los funcionarios romanos tenían buenas razones para creer en el culto de Mithra, porque» «los funcionarios prusianos tenían buenas razones para sentirse atraídos por la masonería, porque»; «los ciudadanos romanos tenían buenas razones para ser politeístas, porque», etc. 
heim ${ }^{7}$, bordando aquí uno de esos ejercicios de análisis de texto tan suyos, a la vez que reconstruye cada una de las cuestiones que hilan su propio argumento. Como el fragmento no tiene desperdicio, invito a la lectura del texto y aquí, a manera de postulados, sólo enumero los puntos centrales de la argumentación. Puestos en un lenguaje que servirá a esta exposición, básicamente éstos podrían ser los siguientes:

- Para Durkheim, el teórico de la magia debe tomar en consideración al menos dos cosas: (1) que, sensatamente, las mentes - de primitivos o desarrollados - no pueden estar diferentemente diseñadas. Y (2) que, por supuesto, los inputs informativos y los recursos cognitivos disponibles a ambos pueden ser, y de hecho son, efectivamente distintos.

- Por la propia fuerza de las cosas, el primitivo durkheimiano que cree en la magia no dispone de los eficaces ejemplares del razonamiento metódico, ni de las sofisticadas teorías que después de siglos han cristalizado en la ciencia occidental.

- Naturalmente, como apunta Boudon en su comentario, un sujeto que no conoce las leyes de la transformación de la energía tendrá mayores dificultades para distinguir entre producir el fuego y producir la lluvia que quien las conoce.

- Pero, muchas de las actividades de los primitivos de Durkheim, tales como la pesca o la agricultura, revisten gran complejidad y requieren algo más que arrojo y fuerza bruta. Exigen imaginación, pericia y — renovadosrecursos cognitivos. En sus condensaciones, estas «teorías» (o si se quiere prototeorías) encierran ricos tesoros de ejemplares de procedimiento, además de secretos no sólo técnicos sino de — plausible o verosímil- asociación causal.

— En este sentido laxo, las «teorías» no pueden verse, pues, como monopolio de la civilización occidental. A su manera, también los individuos de las sociedades primitivas tienen las suyas. Y, por muy simples que éstas sean, también ellos conciben sistemas de relaciones que explican bien el crecimiento de las plantas, o los intercambios entre la planta y el suelo, o la influencia del hombre en la producción agrícola, etc.

- Al igual que en nuestras sociedades altamente tecnificadas y desarrolladas "ciencia» y "visión del mundo» suelen retroalimentarse y legitimarse recíprocamente, constituyendo verdaderos sistemas de creencias teóricas y prácticas, las teorías de los primitivos durkheimianos también se compa- 
decen bien con su concepción religiosa del mundo, la alimentan y la legitiman, a la vez que sacan de ella buena parte de su propia fuerza. En este sentido, Durkheim parece pensar que las creencias mágicas son como una suerte de teorías aplicadas que las sociedades primitivas sacan de las doctrinas religiosas tenidas por verdaderas, exactamente de la misma forma que el saber aplicado de nuestros peritos ingenieros sale de su correspondiente ciencia fundamental.

- Obviamente, los primitivos de Durkheim saben poco o nada acerca de los métodos de verificación en ciencia. No obstante, de forma similar al proceder del conocimiento ordinario y metódico o científico, también ellos buscan - y encuentran - apoyo en corroboraciones estadísticas. Alabando la sutileza del argumento de Durkheim en este punto, Boudon (ibídem: 38) nos recuerda que los rituales destinados a invocar la lluvia suelen practicarse justamente en períodos en los que ésta se asocia a la cosecha y en los que necesariamente buena cosecha y lluvia se sincronizan. Así, con más frecuencia que no, el rito de invocación suele ir acompañado de la llegada efectiva de las lluvias, con lo que la creencia causal recibe el apoyo de la correlación estadística.

- Naturalmente, dice Boudon (ibídem: 39-40) que un sujeto que no domina el arsenal estadístico está peor equipado para validar cualquier relación de inferencia causal y para juzgar si un conjunto de observaciones autoriza o no la aserción " $A$ es la causa de $B$ ». Pero, como tendremos oportunidad de ver más adelante, si se han de dar mecanismos correctores o estar entrenado en técnicas bayesianas para no incurrir en el error - primiti vos o no-, muchos estaríamos expuestos a los mismos fallos. No parece, pues, que se trate de fallos "civilizatorios», sino más bien de deslices o fallos en el razonamiento que pueden afectar a todas las culturas por igual.

- Y, si esto es así, lejos de obedecer a una lógica particular, estos primitivos siguen muy de cerca los mismos procedimientos por los que diariamente nos persuadimos de toda suerte de proposiciones frágiles o inciertas. En este sentido, el mago que cree en la eficacia de los rituales destinados a invocar la lluvia o a favorecer la fertilidad, el hombre moderno que cree en las virtudes de una determinada dieta, o el médico que asocia causalmente depresión clínica y suicidio, no parecen obedecer a lógicas diferentes.

Siempre que este análisis se considere pertinente, «es preciso admitir la existencia de una categoría de razones que, con todo y ser suficientemente bue nas como para tener un efecto sobre las creencias, no están objetivamente fun dadas. Aunque sus creencias mágicas estén desprovistas de fundamento objetivo, el mago tiene buenas razones para creer lo que cree. El problema a resolver es cómo, efectivamente, las razones pueden ser consideradas a la vez 
buenas y no-objetivamente fundadas. Ésta es la cuestión a la que tendrá que enfrentarse la discusión que aparece a continuación.

\section{Tampoco somos tan irracionales: la argumentación contra la tesis de R.A. Shweder}

Iniciada por D. Kahneman y A. Tversky, la tradición de Heuristics and Bia ses ('heurísticas y sesgos') ha aportado interesantes resultados experimentales $y$, en buena medida, ha contribuido a dar un giro sustancial a la psicología cognitiva de las últimas décadas. Lo que esta tradición investigativa ha aportado a través de sus experimentos de laboratorio es la idea de que el razonamiento intuitivo lleva sistemáticamente a respuestas fallidas, siempre que los experimentos involucren cuestiones - no necesariamente complejas - de representatividad estadística, probabilidad, colinearidad y acusación o asociación causal. Lo que ha dado prominencia a estos hallazgos es el alto índice de representatividad de las respuestas fallidas en cualquiera de los experimentos, máxime cuando, precisamente para garantizar el éxito de las pruebas in vitro, éstos se diseñan expresamente de forma tal que excluyan posibles factores distorsionadores (factores afectivos, o de socialización, difu sión o contagio $)^{8}$.

Dado el sorprendente éxito de esta tradición experimental, lo que inicialmente se pensó como un simple procedimiento para el estudio de los procesos cognitivos, con gran rapidez pasó a cristalizarse en tesis generales sobre la naturaleza del razonamiento intuitivo. Como reconocen sus propios impulsores, muy pronto se pasó del método al mensaje (cfr. Kahneman y Tversky, 1982: 124). Así, de las constataciones iniciales sobre desviaciones del juicio intuitivo en un gran número de problemas sencillos que involucraban juicio estadístico y cálculo de probabilidad, se llegó — no sin controversia ${ }^{9}$ - a aseveraciones más o menos extremas según las cuales, sea cual sea la causa, la mente humana se presenta como "víctima de ilusiones» siempre que razona en contextos de incertidumbre o sobre cuestiones de probabilidad (PiatelliPalmarini, 1994), "mal diseñada" para trabajar con el cálculo bayesiano (S.T. Gould, 1999: 469), o "predispuesta a desviaciones sistemáticas de las normas estándar de la racionalidad» (Bazerman y Neadle, 1986).

En el contexto de esta - no por controvertida menos fecunda- tradición investigativa, la idea de Shweder (1977) ya quedaba expresada en el sugerente título de su artículo "Likeliness and likelihood in Everyday Thought: Magical Thinking in Judgements about Personality». En este escrito el autor presentaba un conjunto de distintos experimentos en los que se sometía a los sujetos a emitir juicios en torno a cuestiones que comportaban inferencia estadística. Uno de estos experimentos consistía en presentar a diversos grupos

8. Para una crítica más sólida a estos experimentos por parte de los psicólogos evolucionarios, cfr. Samuels, Stich y Bishop, 2000.

9. Ibídem. 
de estudiantes de la universidad de Harvard una serie de cuestiones dirigidas a estimar, en una muestra de cien personas elegidas al azar, la frecuencia de distribución de dos trazos de personalidad específicos, a saber, la confianza en sí mismo o autoestima y la capacidad de liderar. En este caso, de forma reiterada en los distintos grupos, la estimación subjetiva arrojó una respuesta siempre cercana a la atribución del 65\% de autoestima y del $20 \%$ de atribu tos de liderazgo a los individuos de la muestra.

Acto seguido, se invitaba a los mismos estudiantes a emitir un juicio paralelo sobre la posible relación de influencia del primer rasgo de personalidad (la autoestima) sobre el segundo (la capacidad de liderazgo). Contra todo pronóstico, y en franca contradicción con sus apreciaciones empíricas inmediatamente anteriores, los juicios también convergieron en este punto. La gran mayoría de los interrogados afirmó creer en una clara relación de influencia del primer rasgo sobre el segundo. La autoestima se apreciaba claramente como condición necesaria de la capacidad de liderazgo. Siendo así, la conclusión final a la que llegaban entraba en clara contradicción con sus propias apreciaciones estadísticas:

En el caso de la autoestima y el liderazgo, la mayor parte de los estudiantes americanos afirmaron que saber si alguien tenía o no autoestima les capacitaba para predecir si tal persona era o no un lider. Esta aseveración se hacía a pesar de que contradecía su propia información frecuencial pertinente a la correlación. (Shweder, 1977: 643, énfasis añadido)

Si, siguiendo a Boudon, establecemos la tabla de contingencia correspondiente a esta estimación, se puede ver claramente cuán débil es la correlación inicial entre las dos variables ${ }^{10} \mathrm{y}$, por consiguiente, el escaso fundamento estadístico de su acusación causal.

Tabla 1. Estimación de la correlación de los dos rasgos de la personalidad. Extraída de Boudon (1990: 89)

\begin{tabular}{ccccc}
\hline & Autoestima & Presente & Ausente & Total \\
\hline \multicolumn{1}{c}{ Liderazgo } & & & \\
\multicolumn{2}{c}{ Presente } & 15 & 5 & 20 \\
Ausente & 50 & 30 & 80 \\
Total & 65 & 35 & 100 \\
\hline
\end{tabular}

Según los datos de la tabla, la probabilidad condicional de mostrar capacidad de liderazgo cuando se tiene autoestima equivale a $15 / 65=0,23$, mientras la probabilidad condicional de mostrar tal capacidad en ausencia de autoestima es de 5/35 =0,14. La diferencia entre ambas probabilidades es de 
0,09, demasiado baja como para considerar que la autoestima apunta a un nexo causal con la capacidad liderazgo. Lo que las estimaciones de los estudiantes sobre las frecuencias de los dos rasgos implicaban era, al contrario, una relación de cuasiindependencia entre las dos variables. Así, cuando en el segundo conjunto de respuestas éstos se mostraron convencidos de que la autoestima es un factor relevante en la determinación del liderazgo, estaban sacando conclusiones que en ningún sentido se inferían de sus propias estimaciones. Como en el caso del pensamiento mágico de las culturas tradicionales, el razonamiento ordinario de los estudiantes de Shweder les llevaba a percibir relaciones causales, no sólo donde seguramente no las había, sino donde sus propias apreciaciones parecían implicar exactamente lo contrario.

Fuesen quienes fuesen (estudiantes, médicos o enfermeras, etc.), de forma reiterada todos los sujetos que respondieron a los distintos experimentos presentaron desviaciones similares del razonamiento intuitivo. De tan importante evidencia Shweder, a su vez, llega a la conclusión de que el razonamiento ordinario obedece a otras reglas, reglas distintas de las de la probabilidad bayesiana, y que, en cualquier caso, parecen acercarle más al pensamiento mágico de las culturas tradicionales que a los procedimientos metódicos de la teoría formal de la probabilidad:

El pensamiento mágico es la expresión de una inclinación universal de los adultos normales a sacar lecciones correlacionales de la experiencia, junto con una inclinación universal a buscar conexiones significativas (semejanzas) entre objetos y eventos. La magia no es menos característica de nuestras actividades intelectuales ordinarias que lo es de las prácticas curativas de los azande. (Ibídem: 637)

La interpretación inmediata de esta evidencia experimental le acerca a la estela de pensamiento de Lévi-Bruhl. Es evidente que, por definición, Shweder no puede subscribir teorías que nieguen las creencias mágicas o que vean en ellas simples formas de retórica destinadas a despertar sentimientos (como la de Tambiah 1979)' ni tampoco teorías "calientes» que ignoran los factores cognitivos (como la de Malinowski 1954). Otras interpretaciones de tipo durkheimiano (como la teoría de Lévy-Strauss 1962) ${ }^{11}$ equiparan «magia» y "ciencia», razonamiento ordinario y razonamiento metódico, con lo que entran en contradicción con "sus» evidencias. La alternativa de Shweder, como por lo demás la de muchos otros psicólogos cognitivos, le aproxima —innecesariamente según Boudon- a la tesis del pensamiento prelógico.

Dado el enorme atractivo que sobre Boudon ejercen los resultados de estos experimentos, la interpretación de Shweder le resulta particularmente revulsiva. De hecho, la alternativa le desborda por arriba (en tanto distingue entre

11. TAmbiah, S.J. "Form and meaning of magical acts: a point of view», en HorTON, R ; FinNEGAN (1979) (comp.). MalinowsKi, B. (1954), Magic, Science and Religion, Garden City, Doubleday. LÉvy-Strauss, C. (1962), Le pensée sauvage, París: Plon, 1983. 
lógicas cotidiana y metódica) y por abajo (en tanto que permite la identificación del razonamiento ordinario con el pensamiento prelógico de Lévi-Bruhl). Aunque la interpretación de Shweder le parece excesiva, su propuesta parece poner una gran presión sobre su tesis racionalista de las creencias, quizá, precisamente, por la propia expectativa que en él despiertan estos resultados $y$, en general, los experimentos trabajados por la psicología cognitiva. Así, cuanto más potente le parece el trabajo de Shweder, tanto más se siente llamado a rebatirlo y se propone hacerlo al menos por tres vías básicamente interrelacionadas. En primer lugar, la información frecuencial pertinente a la que alude Shweder suele ir ambiguamente indicada en las preguntas. En segundo lugar, la información frecuencial pertinente generalmente no puede determinarse más que por referencia a un contexto y éste suele ser muy variable. Por último, la persistencia de los fallos detectados no explica el carácter colectivo de las respuestas, no dice nada acerca del consenso expresado sobre una misma idea falsa. Desarrollar estas tres vías de argumentación consumirá aquí el resto de este apartado.

\subsection{Por qué fallan las preguntas}

La cuestión central en este caso es qué tipo de información logra activarse con las preguntas. Puntualmente, qué información logra movilizar en el estudiante que responde a una pregunta como la que se les presentaba en el experimento de Shweder:

En su opinión ¿las personas que muestran capacidad de liderazgo suelen tener confianza en ellas mismas?

Su idea era que una pregunta así enunciada, normalmente debería movilizar en la mente del sujeto que responde a los valores indicados en la tabla de contingencia 1. Pero, contrariamente a lo esperado, dicha información no se moviliza. De haberlo hecho, los sujetos demandados no habrían emitido un juicio de atribución causal contrario a sus propias apreciaciones estadísticas. Así planteado, más que una cuestión de "pensamiento mágico», se trata de un problema referente al tipo de información frecuencial que se moviliza en cada uno de los casos. La reconstrucción hipotética del razonamiento que sigue el interrogado permite hacer a Boudon (1990: 90-97) una bonita disquisición sobre la información frecuencial pertinente que se reproducirá aquí con cierto detalle.

¿Qué tipo de información puede movilizar la pregunta? A simple vista, una cuestión de este tipo no parece pedir otra cosa que determinar si un predica $d o(x)$ se aplica a un sujeto (y). Pero la información necesaria para responderla - positiva o negativamente - no viene determinada por la simple forma de la pregunta. Depende de un contexto variable, con lo que, en muchas ocasiones, la información pertinente pasa a ser conjetural. 
Ahora bien, si se designa por « $\mathrm{x}(\mathrm{y})$ » la proposición que indica que "y es $\mathrm{x}$ », o que $\mathrm{x}$ es un predicado de $\mathrm{y}$,

la información frecuencial pertinente que permitirá responder - positiva o negativamente - a la pregunta " $i x(y)$ ?» es con frecuencia incierta y variable según el contexto, y no siempre es evidente qué tipo de información se le ha de asociar en uno u otro caso.

— Así, en ciertos casos, se subscribirá sin duda la proposición « $x(y)$ » si $x(y)$ aparece como verdadero en todos los casos, es decir, si puede interpretarse como una regularidad analítica o una ley causal. También suele hacerse cuando $x$ se interpreta como un rasgo distintivo de $y$ (Boudon pone aquí el manido ejemplo del «negro» de todos los cuervos). Aunque el enunciado se sabe vulnerable a un único contraejemplo, se tiende a tener a " $x(y)$ » por verdadero (tal es la fuerza evocativa del principio de la inducción) ${ }^{12}$. Para estos casos, la información frecuencial pertinente se puede representar en una tabla de dos entradas en la que a uno de ellos corresponde un efectivo nulo. La descripción exacta de Boudon (ibídem: 90) es:

Tabla 2. Boudon ibídem: 92

\begin{tabular}{lll}
\hline & Negros & No-negros \\
\hline Cuervos & N(todos $)$ & 0 (alguno $)$ \\
\hline
\end{tabular}

El enunciado implica que todos los elementos del conjunto están caracterizados por el mismo valor. Así, si «p $[x(y)] »$ designa la proposición de objetos « $y$ » que presentan la propiedad «x», la información frecuencial pertinente que corresponde a la afirmación « $x(y)$ » es, evidentemente,

$$
p[-x(y)]=0
$$

— Un segundo caso correspondería a una interpretación de « $x(y) »$ como:

Las sonatas para piano de Beethoven son admirablemente interpretadas por Alfred Brendel.

Como en el caso precedente, la información frecuencial pertinente se determina a partir de una tabla con una variable, aunque aquí los parámetros cambian. La proposición será suscrita en el caso de que la interpretación por Brendel de varias sonatas —o alternativamente de muchas de ellas- pueda ser calificada de admirable. En este caso, para el enunciado « $x(y)$ » pue-

12. Boudon suele recoger algunos experimentos de Kahneman y Tversky que resaltan aún más rotundamente cómo, de forma persistente y universal, se suele movilizar implícitamente este principio. Cfr. 1990: 72 y s., en donde lo ilustra en extenso. 
de ser subscrito, la condición que ha de satisfacer la información frecuencial pertinente es:

$$
\mathrm{p}[x(y)]=\text { varias (alternativamente muchas) }
$$

— Puede darse un tercer tipo de interpretación del enunciado « $x(y) »$, tal como:

Los trenes americanos son poco exactos.

Un pasajero que hace uso frecuente de los mismos posiblemente aceptaría esta proposición si tiene evidencias de que de cada diez viajes sólo dos sufren retardo. En este caso, con una única variable a la que corresponde la condición, subscribiría la proposición siempre que la información frecuencial pertinente haga referencia a una desigualdad, así:

$$
x(y) \text { si y sólo si } p[x(y)]>0,2
$$

- Aún podría pensarse en otros casos en los que el enunciado « $x(y)$ » se da en otro contexto o conversación en el que el enunciado se interpreta comparativamente, como cuando se les compara, por ejemplo, con los trenes franceses. En esta ocasión, lo que se querría decir con:

Los trenes americanos son poco exactos.

viene sugerido por el contexto de la conversación, y seguramente lo que quiere decir es que:

Los trenes americanos son menos exactos que los franceses.

Aquí la información frecuencial pertinente para poder aceptar la proposición $x(y)$ debe pensarse en una tabla con cuatro casos:

Tabla 3. Boudon ibídem: 94

\begin{tabular}{lcc}
\hline Trenes & Exactos $(x)$ & Inexactos $(-x)$ \\
\hline Americanos & 2 & 8 \\
Franceses & 1 & 9 \\
\hline
\end{tabular}

En este contexto, la afirmación puede ser aceptada sólo en casos en los que se dé una desigualdad, sea cual sea la diferencia. Aquí,

$$
p[x(y)]-[p x(-y)]=>0,1
$$

Incluso en casos en los que la desigualdad es muy débil, ésta se interpreta como interesante y suficiente para fundamentar la proposición. 
Sin duda existen muchas otras ocasiones en las que la aceptación del enunciado " $x(y)$ » tendría que movilizar una información frecuencial perti nente más rotunda, es decir, en las que implícitamente se exigiría una fuerte correlación. En tales casos, antes de indicar causalidad o afirmar el vínculo causal de un factor con otro, ciertamente habría que asegurarse de que todos los indicios relativos al dicho factor se han tomado en consideración.

De hecho, son muchos los casos en los que, cuando se emite un enunciado del tipo « $x(y)$ » en una conversación cualquiera, los interlocutores lo asocian de forma inmediata y sin ambigüedad a uno de estos tipos (cfr. tabla 5). Pero, en muchas otras situaciones, la información frecuencial requerida, semánticamente muy sensible, resulta ambigua o está subdeterminada.

Así se puede ver cómo, según el contexto conversacional en el que se emite, un mismo enunciado puede ser relacionado a uno u otro tipo de información frecuencial. A veces se acertará y en otras muchas la conjetura sobre el tipo de estructura pertinente puede movilizar la información fre cuencial equivocada o, lo que es lo mismo, puede no movilizar la correcta.

Tabla 4. Boudon (ibídem: 95)

\section{Información frecuencial}

Algunos tipos requerida Ejemplos

Tipo 1 $x(y)$ si $p[x(-y)]=0$

Los cuervos son negros

Tipo 2 $x(y)$ si $p[x(y)]=$ «muchos» Las sonatas de Beethoven

Tipo 3 $x(y)$ si $p[x(y)]-p[x(-y)]>0,1$ Los trenes americanos

Tipo 4 $x(y)$ si $p[x(y)]$ «mucho» más grande que $p[x(-y)]$

La que no movilizó el experimento: Shweder

Tal es para Boudon (ibídem: 97) el caso de los estudiantes del experimento de Shweder. Según la reconstrucción de su razonamiento, la respuesta a la simple pregunta del test implica ante todo que los sujetos del experimento movilicen una proposición de hecho que quepa dentro de la estructura del cuadro, una proposición del tipo:

\section{1. [«frecuentemente, los líderes tienen confianza en ellos mismos»],}

y luego, que (de forma no consciente) la asocien a una proposición del tipo 2, esto es, a:

2.

$$
[« p[x(y)]=« \text { muchos» }] .
$$

Sólo entonces sacarán la conclusión de que:

3. [«los líderes son individuos que tienen confianza en ellos mismos»]. 
Mediante esta simple reconstrucción de su argumentación puede desvelarse el «misterioso» razonamiento que hay detrás de éste y de prácticamente todos los demás experimentos. Sí, efectivamente, en contra de las expectativas de Shweder sus estudiantes no movilizaron una estructura de tipo 4, es seguramente porque, a diferencia de los científicos sociales, ellos no tienen el hábito de pensar que toda proposición « $x(y)$ » debe partir de una información frecuencial de este tipo. Ello no significa que no siguieran reglas legítimas de inferencia. Atribuir el fallo a pensamiento mágico parece una interpretación cuando menos exagerada.

Si Boudon está en lo correcto, en ambos casos, el de Shweder y el de sus estudiantes, los sujetos parecen tener buenas razones para creer lo que creen, aunque en los dos casos las conclusiones a las que llegan son falsas, dudosas o frágiles. Falsas en el caso de las cobayas, puesto que afirman un vínculo o una asociación causal de las variables en contra de su propia evidencia estadística. Dudosa, en el caso de Shweder, puesto que, al presumir que la forma de la pregunta movilizaría de manera biunívoca una información frecuencial de tipo 4, concluye asociando el fallo de razonamiento observado a pensamiento mágico. Para Boudon ambas instancias constituyen excelentes descripciones de lo que él llama el «efecto Simmel» (cfr. 4 a continuación).

\subsection{El "contexto» al que se asocia la pregunta}

Para la argumentación de este punto se echará mano de otro de los experimentos también reproducido por Boudon (1995: 74-78; 1996: 132-136) y que, en lo que respecta a este caso, permite ilustrar mejor el sentido de su crítica.

Ahora se trabaja con una población de expertas enfermeras a las que se pasa un paquete de cien fichas, cada una de las cuales representa a un hipotético paciente. En cada ficha se incluyen dos informaciones, también ficticias, relativas a pacientes que presentan (o no) el sintoma $S$ de la enfermedad y tienen (o no) la enfermedad E. Se pregunta a las enfermeras si, efectivamente, el síntoma $S$ debe ser interpretado como un síntoma claro de la enfermedad $\mathrm{E}$, es decir, si la enfermedad está causalmente vinculada al síntoma.

En la siguiente matriz se reproduce la información compilada que se deriva de las fichas, matriz que, naturalmente, no estuvo disponible a las enfermeras cuando, masivamente, afirmaron un nexo causal entre $\mathbf{E}$ y $\mathbf{S}$.

Tabla 5. Inferencia causal infundada. Extraído de Boudon (1996: 135)

\begin{tabular}{lccc}
\hline & Enfermo & No-enfermo & Total \\
\hline Presente & $37(1)^{*}$ & 33 & 70 \\
Ausente & $17(2)^{*}$ & 13 & 30 \\
Total & 54 & 46 & 100 \\
\hline
\end{tabular}


En la determinación de su respuesta claramente errada, las enfermeras parecieron utilizar sólo una parte de la información, a saber, la proporción de casos (ficticios, aquí 37) en los que el paciente ostentaba a la vez el sinto ma y realmente tenía la enfermedad. Según su intuición, basada en la práctica clínica rutinaria, 37 casos de cada 100 parecen suficientemente representativos como para afirmar el nexo $\mathrm{E} \Rightarrow \mathrm{S}$. Es evidente que esta única información es a todas luces insuficiente para fundamentar una tal relación. De hecho, la información frecuencial pertinente para asertar la existencia de una hipótesis causal apropiada habría exigido la comparación del grupo que presenta la enfermedad y el síntoma con el grupo que no presenta el síntoma pero sí tiene la enfermedad. Referidos a la matriz, los valores de cada uno de estos grupos sería $(1)^{*} 37 / 70=0,53$ y $(2)^{*} 17 / 30=0,57$, respectivamente, con lo que el porcentaje de los enfermos que no presentan el síntoma es mayor que el de los enfermos que sí lo ostentan.

¿Cómo se explica aquí que una correlación ligeramente negativa sea colectivamente percibida como una correlación positiva? En este caso, hay que partir del hecho de que la información frecuencial ha sido ambiguamente transmitida, puesto que se presenta en un formato disperso de fichas en las que, además, se incluyen datos ficticios exagerando ligeramente las cifras normales de incidencia enfermedad-síntoma con las que habitualmente acostumbra a encontrarse la rutina hospitalaria. A diferencia del caso anterior, no puede buscarse una salida válida a la respuesta que, aunque tiende a imponerse mayoritariamente, es clamorosamente falsa.

Boudon (1995: 75-76) lo argumenta en los siguientes términos. Si bien el cuadro de contingencia binaria comprende cuatro informaciones independientes, en la práctica se puede inferir válidamente una acusación causal en casos $(a)$ de una única información, o $(b)$ cuando las distribuciones marginales son muy asimétricas. Si (caso $a$ ), tras un exceso de ingesta de alcohol en dos ocasiones contiguas, observo (o padezco) dos episodios de resaca con fuerte jaqueca, puedo válidamente llegar a la conclusión de que «el alcohol no me sienta bien». Aunque, naturalmente, en este caso no puede determinarse el valor exacto de la correlación, ya que se dispone de pocas indicaciones cualitativas (sólo las dos veces que he tenido jaqueca cuando, de hecho, no soy nada dado a ellas), la certeza del juicio se apoya en el hecho de que la inferencia es correcta. También las enfermeras habrían podido inspirarse en un esquema similar, e incluso con un pequeño número de coincidencias entre $S$ y $E$, podrían haber llegado a acusar válidamente un nexo de tipo $\mathrm{S} \Rightarrow \mathrm{E}$. No obstante, no parece ser el caso. Aunque, seguramente de forma no deliberada o consciente, lo que parecen haber hecho es juzgar que, como por lo demás suele acontecer en todos los fenómenos patológicos a los que están habituadas, la frecuencia relativa de la «enfermedad» es siempre "débil» y la frecuencia de "síntoma» también lo es (caso $b$ ). Pero, como los valores relativos han sido expresamente trucados en las fichas, y las enfermeras no se toman el cuidado de verificar los inputs marginales del test, subvaloran la probabilidad condicional de presentar la enfermedad con y sin el síntoma, res- 
pectivamente, y emiten el juicio erróneo consignado en los resultados del experimento. Si la tabla hubiese sido más ajustada a la realidad, más conforme a lo que en efecto se constata clínicamente, la información según la cual los dos rasgos — enfermedad y síntoma - se encuentran conjugados 37 veces de cada 100 representaría inequivocamente un serio indicio de causalidad (ibídem: 77). Seguro que las enfermeras del experimento siguieron reglas legítimas de inferencia y, aun fallando clamorosamente, seguro que tuvieron sus buenas razones para responder como lo hicieron.

\subsection{Razones «transubjetivas»}

Un último ejemplo servirá para desarrollar este relevante aspecto en la interpretación boudoniana de las creencias colectivas falsas sistemáticamente detectadas en estos experimentos. En este caso el test procede de Casscells y otros. (1978). A diferencia de los anteriores, este experimento tiene importancia práctica y nos será útil más adelante para otras observaciones en el desenlace del ejercicio.

En este caso, el protocolo experimental va dirigido a una audiencia de médicos a la que se le plantea la siguiente pregunta:

¿Si un test para detectar una enfermedad cuya prevalencia es de 1/1000 tiene una tasa positiva de 5\%, ¿qué probabilidad cree que tiene una persona que ha dado positivo de tener realmente la enfermedad, ello en el supuesto de que usted no sabe nada acerca de los síntomas o signos que presenta dicha persona?__ $\%{ }^{13}$.

En la distribución de los resultados, solo $18 \%$ de las respuestas fueron correctas. Un significativo $45 \%$ de ellas ignoró la información relativa a la tasa de referencia y atribuyó a la persona evocada en la pregunta una probabilidad de 0,95. La mayor parte de las respuestas de este distinguido público fue equivocada, y los más se alejaron claramente del cálculo racional correcto.

Tal distribución de las respuestas resulta interesante, al menos por dos razones. En primer lugar, porque las respuestas aparecen muy estructuradas y caen en un valor también muy específico. En efecto, la moda es muy alta y la gran mayoría de los médicos sostiene que el individuo al que alude la pregunta tiene una probabilidad de 0,95 de estar realmente enfermo. Por otra parte, la respuesta más representada está muy lejos de ser verdadera. De hecho, únicamente el $18 \%$ de los médicos da con la respuesta correcta. El experimento ha generado una auténtica creencia colectiva falsa, no ya sólo en un sentido agregado, sino en otro sentido aún más importante, a saber, es resultado de la aplicación de un mismo esquema de razonamiento. De hecho,

13. "If a test to detect a disease, whose prevalence is $1 / 1000$, has a false positive rate of 5 percent, what is the chance that a person found to have a positive result actually has the disease, assuming you know nothing about the person's symptoms». 
todos los que dieron por resultado 0,95 tuvieron que seguir el mismo procedimiento que Boudon (ibídem: 131) reconstruye más o menos literalmente en los siguientes términos. En la pregunta, los médicos entendieron exactamente lo que quería decir un "falso positivo». En cambio, no comprendieron tan bien lo que quería sugerir el término «tasa» referido a los «falsos positivos» (false positive rate). En realidad, no estaban seguros del denominador al que debía referirse el número de "falsos positivos», sí al de los «verdaderos positivos», o más bien, al de los «verdaderos negativos». Siempre resulta más sencillo responder atendiendo al primero de ellos. No obstante, la noción misma de test crea un contexto comunicativo en el que se ha de buscar la "palabra clave» y, cualquiera que se lo tome en serio, tendrá la tendencia a responder con una probabilidad significativa, es decir, lo más alta posible para que la prueba tenga sentido ${ }^{14}$. Así, aunque no de forma consciente, el argumento que mentalmente todos ellos se arman al responder puede bien ser el siguiente:

(1) Como un test ha de ser válido por definición, la probabilidad que se me pregunta debe ser cercana a 1 .

(2) Por otra parte, no se me dice a qué denominador debo referir el número de falsos positivos.

(3) Es más sencillo referir el falso positivo al verdadero positivo que al verdadero negativo.

(4) $\mathrm{Al}$ referir el falso positivo al verdadero positivo se genera un valor de probabilidad alto.

(5) Los médicos concluyeron así como concluyeron. Con seguridad les sobraban razones para hacerlo.

Expresado numéricamente, el razonamiento que supuestamente siguieron les llevaría a lo siguiente:

Si T es el número positivo por cada 100,000 personas y E el número de personas enfermas,

$T=E+5 \%(100) \mathrm{E}=105$ de donde se sigue que, ellos.

$\operatorname{Pr}(E / T)=100 / 105=0,95$, que fue lo que respondió el $45 \%$ de

El razonamiento correcto habría sido:

$T=E+5 \%($ no $-E)=100+5 \%(99,900)=5095$

$\operatorname{Pr}(E / T)=100 / 5095=0,02$, que sólo siguió el $18 \%{ }^{15}$.

14. De la misma manera que un teorema falso no es un teorema, un test que no tiene validez ninguna tampoco es un test. Cfr Ryle (edición de 1966, citado por Boudon en ibídem).

15. Cfr. 1996: 131. 
La respuesta, individualmente falsa, tiende a imponerse a cada uno de los médicos como «la» estrategia de solución al test. En este caso es evidente que la creencia falsa puede resultar de un fallo inteligible y que no responde a razones arbitrarias. De hecho, aunque en éste (y otros casos) las razones no estén objetivamente fundadas, tampoco se trata de razones estrictamente «subjeti vas» ya que, como indica su alta representatividad en las distintas pruebas, éstas no obedecen a rasgos idiosincráticos ni a peculiaridades de individuos aislados. Boudon (1996: 129-130) propone llamarlas "transubjetivas», es decir, razones que son percibidas como sólidas, no ya por un individuo en particular, sino por todos o casi todos los individuos de la prueba.

Esta implicación le resulta particularmente instructiva y muy reveladora, ya que la dimensión "transubjetiva» le permite echar luz sobre el carácter colectivo de las creencias falsas y a la vez identificar un mecanismo capaz de dar cuenta de la aparente irracionalidad en función de sólidas razones, y de hacerlo de forma plausible y convincente (Boudon, 1995: 75-76). Además, la evidencia de los experimentos de la psicología cognitiva parece haber brindado a Boudon la ocasión perfecta para explicar cómo, razones no objetivamente fundadas, pueden aun ser sólidas, buenas razones para adherirse a una creencia incierta o falsa.

\section{El «modelo Simmel»}

El mecanismo por el cual nos adherimos a creencias frágiles dudosas o falsas implicaría así un proceso normal de inferencia que, de forma no consciente, se funda sobre supuestos generales que se tienen por válidos o se dan por sentados y que pueden contaminar las conclusiones. A este mecanismo cognitivo capaz de generar creencias colectivas en ideas frágiles, dudosas o falsas le llama Boudon (1990: 103 s.) el «modelo Simmel».

Para Boudon este modelo «emana directamente de la teoría del conocimiento de George Simmel» (de ahí su nombre), quien lo considera como «un modelo característico del pensamiento humano" (ibídem: 104). No es mi objetivo aquí enzarzarme en una discusión filosófica sobre la inferencia natural, cosa que claramente escapa a la intención de este escrito. Lo que de Los problemas de la filosofía de la historia de G. Simmel ${ }^{16}$ se recoge aquí brevemente son los postulados centrales, tal y como los reproduce Boudon en la construcción de su modelo (ibídem: 103-126).

Aunque Simmel se aparta de la versión ortodoxa de los a priori kantianos ${ }^{17}$ :

16. En 1984 Boudon traduce al francés Die Probleme, una obra claramente enmarcada en la discusión neokantiana de los a priori del conocimiento, un libro difícil de abordar y en cuya introducción el traductor ofrece una lectura particularmente centrada en el problema de inducción.

17. Filósofo, sociólogo y pensador «harto original y amigo de caminos poco trillados» (Ferrater Mora, 1951: 676), Simmel tiene su propia idea del modo como se correlacionan el sujeto y el mundo. Se aparta de la idea kantiana de los a priori porque, aunque éstos revisten un cierto grado de universalidad, no son concebidos como invariantes en el tiempo. 
- Retiene la idea de que todas las actividades del conocimiento movilizan a priori y la idea según la cual ciertas proposiciones, "principios» o "hipótesis generales» (en el lenguaje de Simmel los a priori) presentan a su vez la propiedad de ser metaconscientes y decisivos.

- El conocimiento no es nunca una copia de la realidad y siempre supone una intervención activa del sujeto cognoscente.

- Al lado de proposiciones explícitas sobre las cuales razonamos, siempre introducimos proposiciones implícitas que no aparecen directamente en el campo de la consciencia. Así, puede ocurrir que las conclusiones que sacamos de una teoría nos parezcan diferentes si se ponen de manifiesto sus proposiciones implícitas.

- Simmel considera que ésta es la forma en la que procede el conocimiento humano. Ello implica como consecuencia inevitable la posible discordancia entre los razonamientos tal y como nos aparecen y tal y como son en realidad.

- Ahora bien, si los a priori representan un ingrediente indispensable de todas las ciencias, Simmel se ocupa en Die Probleme de los a priori de la «historia».

- Aunque no los identifica (tampoco era su propósito), especifica sus propiedades esenciales. Con frecuencia toman la forma de "cuadros lógicos» implícitos.

- Ahora bien, si estos supuestos hacen posible el conocimiento histórico (y social), también lo fragilizan.

- Esta ambigüedad, una propiedad intrínseca de los a priori simmelianos, hace posible incurrir en el error.

— En razón de su «validez» y «generalidad» intrínsecas y de su carácter implícito, estos principios generales o «cuadros lógicos» (que representan los a priori simmelianos) escapan a la consciencia crítica.

- Lo que no necesariamente implica que los postulados explícitos escapen a la cautela científica.

- Los supuestos implícitos pueden así distorsionar las conclusiones de cualquier inferencia válida.

- Así, sugiere Simmel, el funcionamiento normal del conocimiento puede conducir al error.

Boudon interpreta la filosofía del conocimiento de Simmel como «una teoría de la inferencia natural, de sus logros y de sus yerros» (ibídem: 129) y , a la luz de sus postulados centrales, reconstruye el mecanismo cognitivo propuesto. En el «modelo Simmel» pretende reconstruir procesos elementales de la inferencia natural que incorporan supuestos implícitos que se tienen por válidos y se dan por sentados, y que permiten comprender cómo puede darse una adhesión «racional» a creencias colectivas frágiles, dudosas o falsas.

Todos los experimentos de la psicología cognitiva con los que trabaja son descripciones típicas de «efectos Simmel». En cada uno de ellos, para un colectivo de individuos que sigue reglas legítimas de inferencia y observa un 
conjunto de protocolos de observación correctos, la intervención de ciertos supuestos (que gozan de validez muy extendida y se dan por sentados) disparados bien por la forma de las preguntas o por el contexto mismo del experimento, sistemáticamente y de forma masiva derivan en conclusiones o juicios inciertos o falsos. Si se hacen aflorar los supuestos implícitos detrás del razonamiento expreso, se comprenden las razones que tuvieron los individuos de la muestra para responder como respondieron. Al margen de estos ejemplos in vitro, Boudon ha ilustrado con eficacia otras descripciones del modelo provenientes de la filosofía y la historia de la ciencia (1990: 129-176), de la sociología del conocimiento (1996: 130-142) y de la sociología moral y política (ibídem: 142-146).

\section{Esbozo de una teoría racional de las creencias}

Un sencillo procedimiento general permitiría, según Boudon, distinguir entre teorías de las creencias fundadas sobre razones (tipo 1) y las teorías «irracionales» (causas distintas de razones) de tipo 2 y 3 . Toda teoría que pretenda explicar racionalmente las creencias ha de poder enunciarlas como resultado de razones: "el sujeto $X$ tiene "buenas razones» para creer $Y$, porque...». Las teorías de tipo 2 y 3 , en cambio, responderían a un enunciado de las creencias del siguiente tenor: «el sujeto $X$ no tiene (o tenía) razones reales de creer $Y$, pero...» (1990: 71).

En algunos casos, la explicación de una creencia o la adhesión a un juicio del tipo "Creo que $X$ » apenas si pone problemas. "Creo que dos y dos son cuatro porque» dos y dos suman cuatro. "Creo que el libro está sobre la mesa, porque», efectivamente, el libro está sobre la mesa. Aquí las creencias están claramente fundadas sobre razones objetivas (1995: 19-20). El acuerdo en este punto apenas si levanta controversia. Los problemas de la propuesta comienzan tan pronto como se abandona la zona segura de lo indiscutible. ¿Cómo dar cuenta en función de razones de creencias positivas falsas? ¿Cómo explicar la adhesión a las creencias falsas colectivas? Las creencias positivas falsas - nos dice Boudon- no son en modo alguno exclusivas del conocimiento ordinario. De hecho, la historia de la ciencia es rica en ejemplos de creencias que en su momento conmocionaron a la comunidad científica y que han acabado siendo falsas. Sin gran controversia, se suelen admitir las buenas razones que tuvieron los científicos en su momento para adherirse a teorías falsas como la del éter, el flogisto o de la «materia cadavérica». ¿Es éste también razonamiento mágico, mentalidad primitiva? Más bien se ha de interpretar como resultado de un mecanismo cognitivo común al razonamiento humano, sea ordinario o científico, del tipo apenas propuesto.

Esta adhesión a creencias falsas cobra verdadera importancia cuando se trata de creencias colectivas. Explicar las creencias de los azande de Evans-Pritchard, o, en general, los ritos de invocación de la lluvia, de iniciación, de fecundidad, u otros característicos de los pueblos tradicionales pone un verdadero reto a cualquier intento de teoría racional de las creencias. El problema 
reviste particular importancia para el sociólogo y desde los clásicos ha estado en la agenda de los problemas irresueltos dejados de lado por la tradición de la teoría económica estándar, en general ciega a la cuestión de las creencias.

A propósito de las creencias mágicas, las creencias colectivas falsas por excelencia, ya se esbozó la teoría durkheimiana de tipo 1. En ella vio Boudon (1990: 39-43) no sólo un legítimo y sólido precedente clásico, sino tambien un claro testimonio de las ventajas asociadas a este paso. Siempre que es posible darlo - dice- «se experimenta un claro sentimiento de progreso». En el caso de la teoría de las creencias mágicas de Durkheim este efecto positivo es más que evidente. Su interpretación supera los defectos de teorías rivales en alza como la de Lévi-Bruhl. De hecho, la interpretación en clave de razones:

- Logra evitar una hipótesis psicológica tan pesada como la de las lógicas variantes o particulares.

- Permite comprender mejor la distribución del fenómeno de la magia en el espacio y en el tiempo.

- Da cuenta de la relación entre prácticas mágicas y tipo de actividades productivas o cosmovisiones integradas a sistemas específicos de creencias religiosas.

- Supera teóricamente a la lévi-bruhliana, una teoría ad hoc, puesto que su noción explicativa medular - la mentalidad primitiva - se introduce puntualmente con el fin de dar cuenta de los mismos fenómenos que explica, y tautológica, en tanto explica la confusión que los primitivos hacen de asociaciones de semejanza y causalidad por su propia tendencia a confundirlas. Tendencia por otra parte garantizada por el hecho mismo de que efectivamente las confunden (ibídem: 41-43).

Las creencias falsas colectivas no son cosa de magia o de primitivos. También permean el espacio de todas las vidas cotidianas, y los experimentos de la psicología cognitiva han sido fecundos en la explicitación de tales evidencias. ¿Por qué los médicos erróneamente creen en una tasa de 0,95 de incidencia? ¿Por qué las enfermeras ven causas donde no las hay? La transcripción de estas creencias colectivas falsas a razones requirió la introducción de un mecanismo cognitivo para hacerlas inteligibles. R. Boudon resolvió esta cuestión con astucia y eficacia metodológica mediante la evocación de los supuestos implícitos transubjetivos, que, una vez explicitados, permiten hacer inteligibles las razones que llevan a la adhesión a creencias inciertas o falsas. Con ello, el propósito de L'Art de se persuader quedaba suficientemente servido (cuadro 2).

Más allá de las creencias positivas, en Le juste et le vrai. Études sur l'objecti vité des valeurs, Boudon (1995) se enfrenta a una cuestión mucho más difícil y controvertida, a saber, al intento paralelo de transcribir también las creencias normativas y los juicios de valor al lenguaje de «razones». En este escrito apenas si podremos plantear el tema, una cuestión en sí misma mucho más pro- 
Cuadro 2. Transcripción de explicaciones de tipo 2 a explicaciones de tipo 1: núcleo central de la propuesta de R. Boudon (1990) sobre las creencias positivas

\section{Factores que} «explican"

«Razones» son causas

Causas que «no-razones»

Tipo de explicaciones

\begin{tabular}{|c|c|}
\hline Factores cognitivos: & 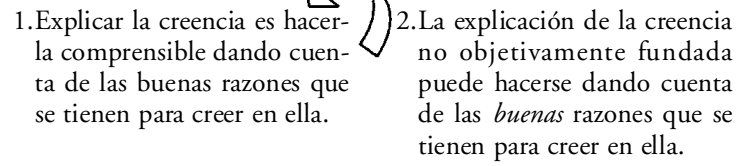 \\
\hline
\end{tabular}

Factores no-cognitivos

4.Las razones que se da el sujeto son tributarias de $(a)$ causas culturales o $(b)$ biológicas evolucionarias.
3. Causas afectivas (accesibles o no a la observación).

blemática, extensa y demandante que la que acabamos de dejar. No obstante, se introducirán algunas notas para poder dar una visión de conjunto y enunciar el esbozo de teoría cognitivista de las creencias que el autor propone.

Aquí, la explicación se enfrenta a creencias normativas y juicios de valor, creencias no asertivas de verdad o falsedad y en principio indemostrables. Concernientes al deber ser, estas creencias serían del tipo " $X$ es bueno» o "X es justo", que, en términos del autor, se expresan en enunciados "axiológicos» (ibídem: 22). El empeño de su transcripción a «razones» resultará mucho más complicado y presupone un esfuerzo mayor y bastante más audaz. ¿Cómo, o sobre qué sustentar este nuevo salto? ¿Son susceptibles las creencias normativas y los juicios de valor de una transcripción del tipo "Creo que $X$ es bueno... porque», "Creo que $X$ es justo... porque»?

Boudon (1995) da aquí un sorprendente giro de timón y rompe fuego con una batería interesante de cuestiones con las que arranca su introducción de Le juste. ¿Por qué, se pregunta, desde el público más llano hasta las más altas esferas de la opinión, la petición del colegio médico francés de indulto de sus colegas involucrados en el escándalo de la sangre contaminada ha producido una indignación tan grande en todos los estamentos sociales y en todos los horizontes de opinión? Si todas las loterías que conocemos se montan sobre una idea de retribución —contribución que, normalmente, estimamos condenable, ¿por qué en este caso no nos resulta chocante? ¿Por qué ciertas desigualdades en la distribución social nos resultan tan indoloras? ¿Por qué, en cambio, otras nos resultan intolerables? De hecho nos tomamos con más naturalidad y sosiego la considerable desigualdad en la remuneración del trabajo femenino, o la radicalmente distinta valoración y reconocimiento social de las diferentes capacitaciones profesionales, mientras reaccionamos con enorme indignación frente al listillo que se cuela en la cola de la ventanilla 
de la oficina de administración. ¿Cuál es el origen de estos sentimientos colectivos? (ibídem: 15-19).

Para responder a este reto autoimpuesto, una forma económica de aproximarnos compactamente a los intentos de solución que busca el autor sería fijar la atención en el cuadro inicial de la tipología de teorías. La tradición heredada en este punto se concentra claramente en la vertiente irracionalista (causas distintas de razones). Significativamente aquí, la teoría de los resi duos de Pareto (teoría de tipo 3) ve en el origen de estos sentimientos colectivos una mera función racionalizadora que satisface la necesidad psicológica de justificación de nuestras creencias y acciones. La búsqueda de esta función latente no reconocida conscientemente, de estos «significados profundos», ha sido recogida en esta tradición por teorías como la psicoanalítica de $S$. Freud, o, interpretada de forma natural, por otras como la crítica marxista de la ideología. Como ya se ha mencionado, la tradición heredada de la filosofía, la psicología y la sociología casi mayoritariamente se ha decantado por uno $\mathrm{u}$ otro tipo de teoría irracionalista de los valores.

Las teorías racionalistas de tipo 1 (celda 1), notoriamente la teoría de la decisión racional en su versión estándar, básicamente subscriben la racionalidad de los medios, enfrentándose con ello a grandes problemas de demarcación entre creencias positivas y creencias normativas. A excepción de juicios tales como "Este cuchillo es bueno», es decir, "este cuchillo corta y, por tanto, cumple la función de cortar», o "Tal es la buena manera de hacer este puente» = «si no, se corre el riesgo de hundimiento", juicios de valor que claramente encubren juicios de hecho y están próximos a la apreciación de los medios, las creencias normativas no pueden explicarse más que de forma consecuencialista. Aún así, los problemas persisten, pues la indispensable distinción entre medios y fines exigida para sostener tal racionalidad se enfrenta a las dificultades que ponen a la teoría, por ejemplo, fines provisionales o intermedios, fines que a su vez pasan a ser medios en relación con otros fines, etc. Como la frontera entre creencias de tipo 1 y tipo 3 no parece tener visos de resolverse satisfactoriamente por esta vía, la racionalidad instrumental y consecuencialista acaba por resultar demasiado restrictiva para dar cuenta del origen de nuestras intuiciones morales.

El problema de las creencias normativas se presenta con particular agudeza cuando las intuiciones morales adquieren expresión colectiva. Si «en cuestión de gustos no hay disputa», no pasa lo mismo con ciertas intuiciones morales. Si tú prefieres la pesca y yo prefiero la música, puedo imaginar sin ninguna dificultad que tengamos distintas preferencias. Pero no llego a imaginar que tú no experimentes la misma indignación que yo, por ejemplo, frente al abuso de un menor. Las preguntas iniciales con las que Boudon abría su introducción a Le juste iban precisamente en este sentido. En todos los casos citados se evocaban sentimientos colectivos. En este contexto, cuando alguien dice que " $X$ es malo" espera, normalmente, que el otro (generalizado) sostenga la misma creencia. No se trata propiamente de un sentimiento personal. En este caso el sentimiento que se tiene es el de que $X$ es efectivamente malo. Este 
juicio de valor no tiene nada que ver con el juicio de gusto. El sentimiento de certeza tiene aquí una importante dimensión transubjetiva. Se trata de sentimientos colectivos que se viven de modo «objetivo» (Boudon, 1996: 128).

Si para Pareto este sentimiento de "certeza» era una mera racionalización, para Durkheim la certeza del juicio moral no es más que una proyección psíquica de la constricción social, tanto más lograda cuanto menos presente en la consciencia, puesto que, naturalmente, de ser consciente, ésta podría llevar a la insumisión. Nuestra experiencia íntima no parece confirmar tal previsión teórica. Tenemos un sentimiento de certidumbre y no de constricción cuando afirmamos que 2 y 2 son 4 . ¿Por qué las certidumbres morales han de ser de otra naturaleza? (1995: 35).

Para evitar, por una parte, los modelos irracionales y, por otra, escapar de la estrecha versión de la racionalidad instrumental, Boudon (1996: 124) cree que la única alternativa es optar por un nuevo camino. Opta así por un modelo cognitivista que resulte más afín con la dimensión transubjetiva y el sentimiento de certeza moral que acompaña a estas intuiciones colectivamente compartidas. El esbozo de una teoría racional de las creencias lo presenta el autor en los siguientes términos: «Llamo a [mi] modelo [...] el modelo cognitivista, queriendo significar con este adjetivo que dicho modelo asume que las creencias, ya sean de tipo positivo o de tipo normativo, pueden ser derivadas de razones, razones que no pueden reducirse a meras consideraciones de coste-beneficio» (cuadro 3). Como se dijo al comenzar, la propuesta sólo queda sentada y el propio Boudon la presenta como un «esbozo de teoría» (ibídem), esbozo al que hay que reconocerle audacia.

Cuadro 3. Propuesta de transcripción de explicaciones de tipo 3 a explicaciones de tipo 1: núcleo central del esbozo de la teoría «cognitivista» de la racionalidad de R. Boudon (1995/1996)

Factores que
«explican» $\quad$ "Razones» son causas Causas que «no-razone»

Tipo de explicaciones

$\begin{array}{lll}\text { Factores cognitivos } & \begin{array}{l}\text { 1. Explicar la creencia es hacer- } \\ \text { la comprensible dando cuen- } \\ \text { ta de las razones que se tie- } \\ \text { nen para creer en ella. }\end{array} & \begin{array}{l}\text { 2. La explicación de la creencia } \\ \text { no objetivamente fundada } \\ \text { puede hacerse dando cuenta } \\ \text { de las buenas razones que se } \\ \text { tienen para creer en ella. }\end{array}\end{array}$

Factores no-cognitivos

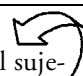

4. Las razones que se da el suje- 3 . to son tributarias de $(a)$ causas culturales o $(b)$ biológicas evolucionarias.
3. "Creer que algo es verdadero, bueno, justo, porque...». Una fundamentación objetiva de los sentimientos morales (la noción de «racionalidad axio lógica»). 


\section{Mejor aún que racional}

El título de este apartado me lo sugiere un bonito artículo de Cosmides y Tooby (1994) «Better than rational: Evolutionary Psychology and the Invisible Hand", que, aparte del atractivo que tiene tal enunciado en el contexto de este escrito, con gran pertinencia apunta a cuestiones que resultan aún más importantes en la discusión del modelo cognitivista hasta aquí expuesto ${ }^{18}$. Este artículo arranca con una anécdota divertida. Cuenta que hace unos años, en un seminario interdisciplinario, mientras economistas, psicólogos y biólogos discutían sobre datos, un economista saltó diciendo: “iYa lo veo! Los datos o son racionales o son psicológicos». La formulación - comentan después de rumiárselo los articulistas - responde a un supuesto tácito que parece sostener la mayor parte de los economistas, a saber, que el comportamiento racional es un estado de naturaleza (ibídem: 327).

Si bien parte del mérito que se le otorgaba en la introducción al esfuerzo de L'Art de se persuader recaía, precisamente, en su talante interdisciplinario $\mathrm{y}$, como hemos visto, Boudon mismo reconoce en distintas ocasiones que los experimentos de los psicólogos cognitivos le brindaron un material decisivo para su proyecto, en el fondo aún le queda alma de economista. En su aproximación a la cuestión del alcance de la racionalidad que tanto y tan legítimamente le ocupa, parece peligrosamente aproximarse al supuesto del «estado natural», aislando de alguna manera su discusión de partes relevantes de la biología, la psicología evolucionaria y del resto de las ciencias de la naturaleza. Incomprensible, y creo desafortunadamente, todas las referencias a la interpretación evolucionaria que muchos psicólogos cognitivos adoptan, o son elusivas o abiertamente descalificatorias. Boudon no parece tomársela seriamente. Las citas a este respecto podrían ser varias, pero he seleccionado una que me resulta particularmente instructiva de su estado de ánimo al respecto. A propósito de los fallos y sesgos del razonamiento que tan cuidadosamente discute en los diversos textos revisados dice, en el artículo en el que presenta el «camino alternativo», o sea, su modelo de racionalidad «cognitivista»:

¿Cómo puede explicarse la existencia de sesgos? Los autores [psicólogos cognitivos] introducen aquí una conjetura filogenética: nuestra intuición acerca de cuestiones estadísticas [habría sido] estructurada en la era temprana de la humanidad como un efecto de [nuestra] experiencia como cazadores y transmitida de generación a generación. Tal estructuración habría tenido el efecto de hacernos poco familiares los procedimientos de la inferencia bayesiana. La conjetura es interesante, pero está sujeta a varias objeciones. Primero, está traída por los pelos y es difícil de probar o refutar —aunque teóricamente

18. El chiste tiene más de una lectura y, naturalmente, el empeño de este escrito no es cubrirlas todas. Uno de los lectores del texto me indicó con puntual pertinencia que lo que aquí se confunden son las razones (epistémicas) que tenemos para creer en algo y las causas (cognitivas o sociológicas) de que creamos. (DOMĖNECH, A. «El eterno retorno de Calicles». Conferencia pronunciada en Málaga, diciembre de 2000). 
falsable, no lo es prácticamente. Por otra parte, ¿cómo es posible explicar qué procedimientos que llevan a predicciones erróneas sean transmitidos sin corrección de una generación a la otra? Generalmente, la teoría evolucionaria enseña que los rasgos mal adaptados se eliminan. Aquí curiosamente se la interpreta como, al contrario, sugiriendo que los retiene en el tiempo. ¿Por qué tendría nuestra intuición geométrica que ser correcta y nuestra intuición estadística, sesgada? [...]. (1996: 130)

Considero, en cambio, que la polémica que mantienen los psicólogos cognitivistas y los psicólogos evolucionarios sobre el alcance de la racionalidad resulta mucho más instructiva que esto. Y también creo que las argumentaciones de nuestro autor contra toda suerte de lógicas variantes o pensamientos prelógicos, o de heurísticas problemáticas que sesgan el razonamiento, o sobre la fijación o adhesión de creencias positivas falsas, o los supuestos transubjetivos y las falsas creencias colectivas, su preocupación por las intuiciones morales compartidas y el sentimiento de certeza moral encontrarían en esta tradición investigativa ricas y sugerentes fuentes, si no de solución sí al menos de inspiración y debate.

\section{Referencias bibliográficas}

Barkes, H.; Hammond, K. (eds.) (1986). Judgement and Desition Making: An Inter disciplinary Reader. Cambridge: Cambridge University Press.

Bazerman, M.; Neale, M. (1986). «Heuristics in negotiation». En: ARKes; HaMMOND (eds.), 1986.

BoudON, R. (1998). Études sur les sociologues classiques. París: Quadrige, PUF.

- (1998a). «Social mechanisms without black boxes». En: HedstrÖM, P.; SwEDERBERG, R. (eds), 1998, p. 172-203.

- (1998b). "Limitations of Rational Theory», American Journal of Sociology, vol. 104, no 3: 817-8828.

- (1996). "The Cognitivist Model". A generalized "Rational-Choice Model"». Rationality and Society, vol. 8, no 3: 123-150.

- (1995). Le Juste et le vrai. Études sur l'objectivité des valeurs et de la connaissance. París: Fayard.

- (1990). L'Art de se persuader des idées douteuses, fragiles ou fausses. París: Fayard.

- (1986). L'ideologie, ou l'origine des idées reçues. París: Fayard. Nueva edición Seuil, Collection Points, 1992.

- (1979). La logique du social. París: Hachette. Nueva edición 1983. Traducción castellana Rialp, Madrid, 1979.

Casscells, W.; SchoenberG, A.; Grayboys, T. (1978). «Interpretation by physicians of clinical laboratory results». New England Journal of Medicine, 299: 999-1000.

Cosmides, L.; Tooby, J. (1994). «Better tah Rational: Evolutionary Psychology and the Invisible Hand». American Economic Review, vol. 84, no 2: 327-332.

Durkheim, E. (1919). Les Formes élémentaires de la vie religieuse. París: PUF, 1960. Traducción castellana en Ariel, Madrid, 1982. 
Evans-Pritchard, E.E. (1937). Witchcraft, Oracles and Magic among the Azande, Oxford: Claredom Press, 1968. Traducción castellana en Crítica, Barcelona, 1993.

Ferrater Mora, J. (1951). Diccionario de Filosofia. Buenos Aires: Editorial Sudamericana, $5^{\text {a }}$ edición, 1965.

Gould, S. (1992). Bully for Brontosaurus. Further Reflections in Natural History. Londres: Penguin Books.

Hedström, P.; Swedberd, R. (eds.) (1998). Social Mechanisms. An Analytical Appro ach to Social Theory. N.Y.: Cambridge University Press.

Horton, R.; Finnegan, R. (comp.) (1973). Modes of Thought. Londres: Faber.

Kahneman, D.; Slovic, P.; TVersky, A. (1982). Judgment under Uncertainty: Heu ristics and Biases. Cambridge: Cambridge University Press.

LÉVI-Bruhl (1922). La Mentalité primitive. París: PUF, 1960.

- (1963). L'Âme primitive. París: PUF.

LÉVY-STRAuSs (1962). Le pensée sauvage. París: Plon, 1983. Traducción castellana en Fondo de Cultura Económica, México, 1964.

Malinowski, B. (1954). Magic, Science and Religion. Garden City: Doubleday. Traducción castellana en Ariel, Barcelona, 1974.

MiLl, J.S. (1974). «On Fallacies». Libro V de A System of Logic, Rationactive and Induc tive. Edición citada: Routledge and Kegan Paul.

PARETO, V. (1909). Escritos sociológicos. Selección, traducción e introducción de María Luz Morán. Madrid: Alianza Editorial, 1987.

Perelman, C.; Olbrechts-Tyteca, L. (1958). Traité de l'argumentation. París: PUF. Piatelli-Palmarini, M. (1994) Inevitable Illusions: How Mistakes of Reason Rule Our Minds. N.Y.: John Wiley \& Sons.

RyLE, G. (1949). The Concept of Mind. Londres: Hutchinson, 1966.

Samuels, R.; STich, S.; Bishop, M. (en prensa). Common Sense, Reasoning and Ratio nality. Vancouver Studies in Cognitive Science, vol. 1.

SHWEDER, R.A. (1977). «Likeliness and Likelihood in Everyday Thought: Magical Thinking in Judgements about Personality». Current Anthropology, 18(4): 437-59.

Simmel, G. (1923, edición citada). Les Problèmes de la Philosophie de l'Histoire. París: Presses Universitaires de France, 1984.

- (1900). Filosofía del dinero. Madrid: Instituto de Estudios Políticos, 1974.

TAMBiaH, S.J. (1973). «Form and meaning of magical acts: a point of view». En: HORTON; FINNEGAN (comp.).

- (1973). p. 230-248.

Toulmin, S. (1958). The Uses of Argument. N.Y./Londres: Cambridge University Press.

TVERSKY, A.; KAHNEMAN, D. (1973) «Availability: a heuristic for judging frequency and probability». Cognitive Psychology, 5: 207-232.

WiLsOn, E.O. (1998). Consilience. La unidad del conocimiento. Barcelona: Galaxia Gutemberg-Círculo de Lectores, 1999. 